\title{
Many-body effective mass and spin susceptibility in a quasi-two-dimensional electron liquid
}

\author{
R. Asgari ${ }^{1}$ and B. Tanatar ${ }^{2}$ \\ ${ }^{1}$ Institute for Studies in Theoretical Physics and Mathematics, Tehran 19395-5531, Iran \\ ${ }^{2}$ Department of Physics, Bilkent University, 06800 Bilkent, Ankara, Turkey
}

(Received 9 March 2006; revised manuscript received 8 May 2006; published 1 August 2006)

\begin{abstract}
We present numerical calculations of the effect of electron-electron interactions on the quasiparticle properties such as the effective mass and the Landé $g$-factor in a GaAs/AlGaAs triangular quantum well from which the spin susceptibility is obtained. For this purpose, we consider quantum many-body effects associated with charge- and spin-density fluctuations induced many-body vertex corrections. The approach is based on the many-body local-field factors which are extracted from Fermi hypernetted-chain self-consistent calculation through the fluctuation-dissipation theorem. We find the spin susceptibility in good agreement with the recent experimental measurements and quantum Monte Carlo simulation data for such a system in the weak and intermediate coupling limits.
\end{abstract}

DOI: 10.1103/PhysRevB.74.075301

PACS number(s): 73.20.Mf, 71.10.Ca

\section{INTRODUCTION}

Two-dimensional (2D) electron systems as realized in semiconductor interfaces are of continuing interest ${ }^{1,2}$ both from basic physics and technological points of view. A great deal of activity was spawned in the last decade to understand the apparent metal-insulator transition (MIT) observed in Si-MOSFET and GaAs based structures. ${ }^{3}$ Although the basic mechanism and the existence of a quantum phase transition is still a matter of on-going debate, experiments have amassed a wealth of data on the transport properties of the 2D electron systems in the metallic state. The experiments $^{4-14}$ are performed on low-density samples where the interaction effects are important and it is becoming clear that to understand the observed behavior realistic modeling of the sample geometry is very important. As the systems invariably have an extension in the perpendicular direction they are, geometrically speaking, quasi-two-dimensional (Q2D) and it is this feature that has to be taken into account in theoretical calculations.

It has been shown ${ }^{4}$ that the resistance of a Si-MOSFET structure increases dramatically by increasing the strength of an in-plane magnetic field, and saturates at a characteristic value of several Teslas. Low-field quantum Shubnikov-de Haas $(\mathrm{SdH})$ measurements on Si-MOSFETs by Okamoto et $a l .{ }^{5}$ revealed that the saturation value is the magnetic field that is necessary to fully polarize the electron spins. An interpretation $^{6,7}$ of the in-plane magnetoresistance in $\mathrm{Si}$ inversion layers suggested a ferromagnetic instability at or very close to the critical density for the Q2D MIT driven by a divergence in the effective mass. Pudalov et al. ${ }^{8}$ have reported direct measurements of effective mass in highmobility Si-MOSFETs over a wide range of carrier density by using a novel technique based on the beating pattern of $\mathrm{SdH}$ oscillations in crossed magnetic fields. These authors measured the effective mass and spin susceptibility in the vicinity of the Q2D MIT, finding no evidence for a divergent behavior but only a moderate enhancement of the effective mass by a factor of $\approx 2-2.5$ over the band mass. Two groups have also reported anomalous density dependence of the modified Landé factor in $n$-doped ${ }^{9}$ and $p$-doped ${ }^{10}$
GaAs/AlGaAs heterojunctions that are in disagreement with results in Si-MOSFETs. The dependence of the spin susceptibility on electron density has been studied by Zhu et al. ${ }^{11}$ who used a Q2D electron gas (EG) of exceedingly high quality. More recently, Tan et al. ${ }^{12}$ performed high precision measurements of the electronic effective mass in Q2D EG over a wide range of electron density. Spin polarization for a Q2D electron system has been studied by a combination of measurements and calculations by Tutuc et al. ${ }^{13}$ Their results revealed the importance of finite thickness of the electron layer and the resulting deformation of the energy surface in the presence of a parallel magnetic field induces an enhancement of the effective mass and Lande $g^{*}$-factor. It is worth mentioning that Vakili et al. ${ }^{14}$ have reported measurements of the effective mass and spin susceptibility in a dilute 2D EG confined to a narrow AlAs quantum well (only $45 \AA$ wide). The electron system investigated in Ref. 14 is quite interesting because the electrons occupy an out-of-plane conduction-band valley, rendering the system similar to $2 \mathrm{D}$ electrons in Si-MOSFETs but with only one valley occupied. The results of Vakili et al. ${ }^{14}$ for spin susceptibility are in good agreement with the QMC results of Attaccalite et al. ${ }^{15}$ even though this simulation has been carried out for a strictly disorder-free EG.

Theoretical calculations of the effective mass and spinsusceptibility of electron systems are performed within the framework of Landau's Fermi liquid theory ${ }^{16}$ whose key ingredient is the quasiparticle concept and its interactions. As applied to the electron gas model this entails the calculation of effective electron-electron interactions which enter the many-body formalism allowing the calculation of various physical properties. A number of calculations considered different variants of the $G W$-approximation for the self-energy ${ }^{17-23}$ from which density, spin-polarization, and temperature dependence of effective mass are obtained. In these calculations the on-shell approximation ${ }^{19,20}$ yields a diverging effective mass but the full solution of Dyson equation yields only a mild enhancement. ${ }^{22,23}$ Other approachesexploiting the similarity to neutral fluid ${ }^{3} \mathrm{He}$ in the vicinity of a MIT found diverging effective mass. ${ }^{24,25}$

In a recent paper De Palo et al. ${ }^{26}$ employed quantum Monte Carlo simulation results for a $2 \mathrm{D}$ electron system in 
conjunction with perturbation theory using the parameters of specific samples of Zhu et al. ${ }^{11}$ to calculate the spin susceptibility and emphasized the importance of Q2D nature of the physical systems. Dharma-wardana in a series of papers ${ }^{27}$ calculated the effective mass, Landé $g^{*}$-factor, and spinsusceptibility for Q2D electron systems within the classicalmap hypernetted chain (CHNC) approximation. He found that the thickness effect on the spin-phase transition provides a clear picture of the changes in the spin susceptibility enhancement leading to a strong increase in the $g^{*}$-factor, while the effective mass is increased from the reduction of the Coulomb potential in thick layers.

The purpose of this paper is to study the quasiparticle properties such as effective mass and spin-susceptibility of Q2D electron systems in the intermediate coupling region in view of the recent experiments of Tan et al. ${ }^{12}$ and Zhu et al. ${ }^{11}$ In a previous work ${ }^{23}$ we studied a strictly $2 \mathrm{D}$ electron system and we calculated the effects of correlations and disorder in the effective mass enhancement. More recently, we concentrated $^{22}$ on Q2D systems, but we had employed localfield factors which were built from the quantum Monte Carlo (QMC) data and were valid for strictly 2D systems. Localfield factors embody correlation effects beyond the random phase approximation and constitute a significant input to our calculations at intermediate couplings. All the experimental samples used in the measurement of transport properties are Q2D in character, therefore to generalize our previous works, we consider a theory in which the layer thickness effects enter the local-field factors. In this direction, we use accurate static structure factors resulting from a Fermi hypernettedchain self-consistent calculation ${ }^{28-30}$ (FHNC) in conjunction with the fluctuation-dissipation theorem to extract the localfield factors which depend on the quantum-well width. We find that for the specific sample parameters of Tan et al. ${ }^{12}$ good agreement between the experimentally observed spinsusceptibility and our theoretical results can be achieved up to the intermediate coupling strength regime. Our results are also in good agreement with the QMC simulations of De Palo $\mathrm{et} \mathrm{al.}{ }^{26}$ in the same range of coupling strengths implying the efficacy of our theoretical approach.

The rest of this paper is organized as follows. In the next section we outline our theoretical approach to calculate the self-energy for Q2D electron systems from which the quasiparticle properties are obtained. The essential ingredients of our theoretical framework are the local-field factors that define the effective electron-electron interactions. We discuss in Sec. III how they are obtained both in the charge and spin channels within the FHNC approximation. Our numerical results for the effective mass, modified Landé $g^{*}$-factor, and spin-susceptibility are presented in Sec. IV where we also make detailed comparisons with experimental data and other theoretical calculations. We conclude in Sec. V with a brief summary of our main results.

\section{THEORY}

We consider a Q2D EG as a model for a system of electronic carriers with band mass $m$ in a semiconductor heterostructure with dielectric constant $\kappa$. We include the effect of thickness of a GaAs heterojunction-insulated gate field-effect transistor (HIGFET) with bare electron-electron interaction $v_{\mathbf{q}}=2 \pi e^{2} F(q d) /(\kappa q)$ which is the Coulomb potential renormalized by the form factor given by

$F(x)=\left(1+\frac{\kappa_{\text {ins }}}{\kappa_{s c}}\right) \frac{8+9 x+3 x^{2}}{16(1+x)^{3}}+\left(1-\frac{\kappa_{\text {ins }}}{\kappa_{s c}}\right) \frac{1}{2(1+x)^{6}}$,

where $d=\left[\hbar \kappa_{s c} /\left(48 \pi m e^{2} n^{*}\right)\right]^{1 / 3}$ and $n^{*}=n_{\text {depl }}+11 n / 32$. Here the depletion layer charge density $n_{\text {depl }}$ is essentially zero and $\kappa_{i n s}=10.9$ and $\kappa_{s c}=12.9$ and $\kappa$ is their average. At zero temperature there are only two relevant parameters for a disorder free, homogeneous Q2D EG in the absence of quantizing magnetic fields, the usual Wigner-Seitz density parameter $r_{s}=\left(\pi n a_{B}^{2}\right)^{-1 / 2}, a_{B}=\hbar^{2} \kappa /\left(m e^{2}\right)$ being the Bohr radius in the medium of interest and the second parameter is the degree of spin polarization $\zeta=\left|n_{\uparrow}-n_{\downarrow}\right| / n$. Here $n_{\sigma}$ is the average density of particles with spin $\sigma=\uparrow, \downarrow$ and $n=n_{\uparrow}+n_{\downarrow}$ is the total average density.

The aim of this section is to provide the theoretical model to calculate the quasiparticle properties of Q2D EG by evaluating the retarded quasiparticle (QP) self-energy $\Sigma_{\text {ret }}(\mathbf{k}, \omega)$ of a paramagnetic Q2D EG and applying the Fermi liquid theory. We emphasize that thickness of a quantum well would be important to consider in a theoretical many-body treatment, since the experiments are performed on samples with varying thickness or specific quantum well geometry. Therefore we will present a self-consistent approach to calculate the spin-symmetric and spin-antisymmetric local-field factors incorporating the quantum well thickness effect.

\section{A. General formulas}

We will employ in our theory the following decomposition for the retarded QP self-energy $\Sigma_{\text {ret }}(\mathbf{k}, \omega)$ :

$$
\Sigma_{\text {ret }}(\mathbf{k}, \omega)=\Sigma_{X}(\mathbf{k})+\Sigma_{\text {line }}(\mathbf{k}, \omega)+\Sigma_{\text {res }}(\mathbf{k}, \omega) .
$$

Here the first term is the Hartree-Fock self-energy. For a 2D EG we have ${ }^{31}$

$$
\Sigma_{\mathrm{X}}(\mathbf{k})= \begin{cases}-2 e^{2} k_{F} E\left(\bar{k}^{2}\right) / \pi & (\bar{k} \leqslant 1), \\ -2 e^{2} k_{F} \bar{k}\left[E\left(1 / \bar{k}^{2}\right)-\left(1-1 / \bar{k}^{2}\right) K\left(1 / \bar{k}^{2}\right)\right] / \pi & (\bar{k}>1),\end{cases}
$$


where $\bar{k}=k / k_{F}$ and $K(x), E(x)$ are complete elliptic integrals of the first and second kind, respectively. The second term in Eq. (2), which is purely real, is given by

$$
\begin{aligned}
\Sigma_{\text {line }}(\mathbf{k}, \omega)= & -\int \frac{d^{2} \mathbf{q}}{(2 \pi)^{2}} v_{\mathbf{q}} \int_{-\infty}^{\infty} \frac{d \Omega}{2 \pi} \\
& \times\left[\frac{1}{\varepsilon(\mathbf{q}, i \Omega)}-1\right] \frac{1}{\omega+i \Omega-\xi_{\mathbf{k}+\mathbf{q}} / \hbar} .
\end{aligned}
$$

Finally, the third term is the so-called "residue" contribution,

$$
\begin{aligned}
\Sigma_{\text {res }}(\mathbf{k}, \omega)= & \int \frac{d^{2} \mathbf{q}}{(2 \pi)^{2}} v_{\mathbf{q}}\left[\frac{1}{\varepsilon\left(\mathbf{q}, \omega-\xi_{\mathbf{k}+\mathbf{q}} / \hbar\right)}-1\right] \\
& \times\left[\Theta\left(\omega-\xi_{\mathbf{k}+\mathbf{q}} / \hbar\right)-\Theta\left(-\xi_{\mathbf{k}+\mathbf{q}} / \hbar\right)\right] .
\end{aligned}
$$

Here $\Theta(x)$ is the step function and $\xi_{\mathbf{k}}=\varepsilon_{\mathbf{k}}-\varepsilon_{F}$ where $\varepsilon_{\mathbf{k}}$ $=\hbar^{2} \mathbf{k}^{2} /(2 m)$ is the single-particle energy with $\varepsilon_{F}$ $=\hbar^{2} k_{F}^{2} /(2 m)$ and $k_{F}=\left(2 \pi n_{2 \mathrm{D}}\right)^{1 / 2}=\sqrt{2} /\left(r_{s} a_{B}\right)$, respectively, being the Fermi energy and wave number.

In Eqs. (4) and (5) $\varepsilon(\mathbf{q}, \omega)$ is a screening dielectric function originating from the effective Kukkonen-Overhauser interaction, ${ }^{18,32}$

$$
\begin{aligned}
\frac{1}{\varepsilon(\mathbf{q}, \omega)}= & 1+v_{\mathbf{q}}\left[1-G_{+}(\mathbf{q}, \omega)\right]^{2} \chi_{\mathrm{C}}(\mathbf{q}, \omega) \\
& +3 v_{\mathbf{q}} G_{-}^{2}(\mathbf{q}, \omega) \chi_{\mathrm{S}}(\mathbf{q}, \omega) .
\end{aligned}
$$

In this expression $\chi_{\mathrm{C}}(\mathbf{q}, \omega)$ and $\chi_{\mathrm{S}}(\mathbf{q}, \omega)$ represent the charge-charge and spin-spin response functions, which in turn define and are determined by the spin-symmetric and spin-antisymmetric local-field factors $G_{+}(\mathbf{q}, \omega)$ and $G_{-}(\mathbf{q}, \omega)$ via the relations

$$
\chi_{\mathrm{C}}(\mathbf{q}, \omega)=\frac{\chi_{0}(\mathbf{q}, \omega)}{1-v_{\mathbf{q}}\left[1-G_{+}(\mathbf{q}, \omega)\right] \chi_{0}(\mathbf{q}, \omega)},
$$

and

$$
\chi_{\mathrm{S}}(\mathbf{q}, \omega)=\frac{\chi_{0}(\mathbf{q}, \omega)}{1+v_{\mathbf{q}} G_{-}(\mathbf{q}, \omega) \chi_{0}(\mathbf{q}, \omega)},
$$

where $\chi_{0}(\mathbf{q}, \omega)$ is the Lindhard response function of a noninteracting 2D EG. ${ }^{33}$ In the paramagnetic electron liquid $G_{ \pm}(\mathbf{q}, \omega)=\left[G_{\uparrow \uparrow}(\mathbf{q}, \omega) \pm G_{\uparrow \downarrow}(\mathbf{q}, \omega)\right] / 2$, where $G_{\sigma \sigma^{\prime}}(\mathbf{q}, \omega)$ are the spin-resolved local-field factors. It is evident from the above equations setting $G_{ \pm}(q, \omega)=0$ we recover the standard random phase approximation (RPA). Although there has been some recent progress in developing wave vector and frequency dependent local-field factors ${ }^{34,35}$ in most applications to date the frequency independent, static local field factors are used. In what follows, we shall make the common approximation of neglecting their frequency dependence.

Quite generally, once the QP self-energy is known, the QP excitation energy $\delta \mathcal{E}_{\mathrm{QP}}(\mathbf{k})$, which is the $\mathrm{QP}$ energy measured from the chemical potential $\mu$ of the interacting EG, can be calculated by solving self-consistently the Dyson equation

$$
\delta \mathcal{E}_{\mathrm{QP}}(\mathbf{k})=\xi_{\mathbf{k}}+\left.\operatorname{Re} \Sigma_{\text {ret }}^{\mathrm{R}}(\mathbf{k}, \omega)\right|_{\omega=\delta \mathcal{E}_{\mathrm{QP}}(\mathbf{k}) / \hbar},
$$

where $\operatorname{Re} \Sigma_{\text {ret }}^{\mathrm{R}}(\mathbf{k}, \omega)=\operatorname{Re} \Sigma_{\text {ret }}(\mathbf{k}, \omega)-\Sigma_{\text {ret }}\left(k_{F}, 0\right)$. For later purposes we introduce at this point the so-called on-shell approximation (OSA). This amounts to approximating the QP excitation energy by calculating $\operatorname{Re} \sum_{\text {ret }}^{\mathrm{R}}(\mathbf{k}, \omega)$ in Eq. (9) at the frequency $\omega=\xi_{\mathbf{k}} / \hbar$ corresponding to the single-particle energy, that is

$$
\delta \mathcal{E}_{\mathrm{QP}}(\mathbf{k}) \simeq \xi_{\mathbf{k}}+\left.\operatorname{Re} \Sigma_{\mathrm{ret}}^{\mathrm{R}}(\mathbf{k}, \omega)\right|_{\omega=\xi_{\mathbf{k}} / \hbar}
$$

Once the QP excitation energy is known, the effective mass $m^{*}(k)$ can be calculated by means of the relationship

$$
\frac{1}{m^{*}(k)}=\frac{1}{\hbar^{2} k} \frac{d \delta \mathcal{E}_{\mathrm{QP}}(k)}{d k} .
$$

Evaluating the $m^{*}(k)$ at $k=k_{F}$, one gets the QP effective mass at the Fermi surface. We remark that the QP excitation energy may be calculated either by solving self-consistently the Dyson equation [Eq. (9)] or using the OSA in Eq. (10). In what follows the identity

$$
\begin{aligned}
\frac{d \operatorname{Re} \sum_{\mathrm{ret}}^{\mathrm{R}}[k, \omega(k)]}{d k}= & \left.\partial_{k} \operatorname{Re} \Sigma_{\mathrm{ret}}^{\mathrm{R}}(k, \omega)\right|_{\omega=\omega(k)} \\
& +\left.\partial_{\omega} \operatorname{Re} \Sigma_{\mathrm{ret}}^{\mathrm{R}}(k, \omega)\right|_{\omega=\omega(k)} \frac{d \omega(k)}{d k}
\end{aligned}
$$

will be used, $\omega(k)$ being an arbitrary function of $k$. Using Eqs. (11) and (12) with $\omega(k)=\delta \mathcal{E}_{\mathrm{QP}}(k) / \hbar$ we find that the QP effective mass $m_{\mathrm{D}}^{*}$ calculated within the Dyson scheme is given by

$$
\frac{m_{\mathrm{D}}^{*}}{m}=\frac{Z^{-1}}{1+\left.\left(m / \hbar^{2} k_{F}\right) \partial_{k} \operatorname{Re} \Sigma_{\mathrm{ret}}^{\mathrm{R}}(k, \omega)\right|_{k=k_{F}, \omega=0}} .
$$

The renormalization constant $Z$ that measures the discontinuity of the momentum distribution at $k=k_{F}$ is given by

$$
Z=\frac{1}{1-\left.\hbar^{-1} \partial_{\omega} \operatorname{Re} \sum_{\mathrm{ret}}^{\mathrm{R}}(k, \omega)\right|_{k=k_{F}, \omega=0}} .
$$

The normal Fermi-liquid assumption, $0<Z \leqslant 1$, implies that $\left.\partial_{\omega} \operatorname{Re} \sum_{\text {ret }}^{\mathrm{R}}(k, \omega)\right|_{k=k_{F}, \omega=0} \leqslant 0$. Thus we see that the effective mass $m_{\mathrm{D}}^{*}$ can diverge at a finite value of $r_{s}$ by one of two mechanisms: ${ }^{22}$ (i) the partial derivative of $\Sigma_{\text {ret }}^{R}$ with respect to $\omega,\left.\quad \partial_{\omega} \operatorname{Re} \sum_{\text {ret }}^{\mathrm{R}}(k, \omega)\right|_{k=k_{F}, \omega=0}$ going to minus infinity at some finite value of $r_{s}$; and (ii) the partial derivative of $\sum_{\text {ret }}^{\mathrm{R}}$ with respect to $k,\left.\quad \partial_{k} \operatorname{Re} \sum_{\text {ret }}^{\mathrm{R}}(k, \omega)\right|_{k=k_{F}, \omega=0}$ going to $-\hbar^{2} k_{F} / m$ at some finite value of $r_{s}$. On the other hand, using Eqs. (11) and (12) with $\omega(k)=\xi_{\mathbf{k}} / \hbar$ we find that the QP effective mass $m_{\mathrm{OSA}}^{*}$ within the OSA is given by 


$$
\frac{m_{\mathrm{OSA}}^{*}}{m}=\frac{1}{1+\left.\left(m / \hbar^{2} k_{F}\right) \partial_{k} \operatorname{Re} \sum_{\mathrm{ret}}^{\mathrm{R}}(k, \omega)\right|_{k=k_{F}, \omega=0}+\left.\hbar^{-1} \partial_{\omega} \operatorname{Re} \sum_{\mathrm{ret}}^{\mathrm{R}}(k, \omega)\right|_{k=k_{F}, \omega=0}} .
$$

Evidently, Eq. (15) is a valid expression for the QP effective mass only in the weak coupling limit. ${ }^{22}$

\section{B. Landau Fermi liquid theory of the various physical properties}

Among the theoretical methods designed to deal with the intermediate density regime, of particular interest for its physical appeal and elegance is Landau's phenomenological theory ${ }^{16}$ dealing with low-lying excitations in a Fermi-liquid. Landau called such single-particle excitations quasiparticles (QPs) and postulated a one-to-one correspondence between them and the excited states of a noninteracting Fermi gas. He wrote the excitation energy of the Fermi liquid in terms of the energies of the QPs and of their effective interactions. The QP-QP interaction function can in turn be used to obtain various physical properties of the system and can be parametrized in terms of experimentally measurable data. Starting with the quasiparticle energy and its relation to the Landau interaction function, one can drive the following relation ${ }^{18}$ for a modified Landé $g^{*}$-factor

$$
\frac{g^{*}}{g}=\frac{1}{1-g_{1}+g_{2}},
$$

where

$$
\begin{aligned}
g_{1}= & m^{*} \int_{0}^{2 \pi} \frac{d \phi}{(2 \pi)^{2}} v(\mathbf{k}-\mathbf{p})\left[1+v(\mathbf{k}-\mathbf{p})\left\{\left(1-G_{+}(\mathbf{k}-\mathbf{p})\right)^{2}\right.\right. \\
& \left.\left.\times \chi_{C}(\mathbf{k}-\mathbf{p}, 0)-G_{-}^{2}(\mathbf{k}-\mathbf{p}) \chi_{S}(\mathbf{k}-\mathbf{p}, 0)\right\}\right],
\end{aligned}
$$

and

$$
\begin{aligned}
g_{2}= & \frac{2 m^{*} m}{\pi^{3}} \int_{0}^{\infty} d z \int_{0}^{\infty} d u v(q)^{2}\left[\frac{\left[1-G_{+}(q)\right] G_{-}(q)}{Q_{-}(q, i \omega) Q_{+}(q, i \omega)} P_{+}(z, u)\right. \\
& \left.+\frac{G_{-}^{2}(q)}{Q_{-}^{2}(q, i \omega)} P_{-}(z, u)\right] .
\end{aligned}
$$

Here $|\mathbf{k}|=|\mathbf{P}|=\mathrm{k}_{\mathbf{F}}$ and $\phi$ is the angle between them, $q=2 z k_{F}$ and $\quad \omega=2 k_{F}^{2} u z / m_{b}$. Furthermore, $\quad Q_{ \pm}(q, i \omega)$ $=\chi_{0}(q, i \omega) / \chi_{C, S}(q, i \omega)$ and the function

$$
P_{ \pm}(z, u)=\frac{\left[\left(z^{2}-u^{2}-1\right)^{2}+(2 z u)^{2}\right]^{1 / 2} \pm\left(z^{2}-u^{2}-1\right)}{\left[\left(z^{2}-u^{2}-1\right)^{2}+(2 z u)^{2}\right]} .
$$

Once the QP effective mass $m^{*}$ and modified Landé $g^{*}$-factor have been calculated the spin susceptibility is found by the following exact relationship:

$$
\frac{\chi^{*}}{\chi_{0}}=\frac{m^{*}}{m} \frac{g^{*}}{g},
$$

where $\chi_{0}$ is the Pauli spin susceptibility.

\section{LOCAL-FIELD FACTORS}

As is clear from Eqs. (6)-(8), (17), and (18) the local-field factors are the fundamental quantities for an evaluation of quasiparticle properties. In this section we introduce the static values of these functions. Our strategy follows that of Ref. 36 which uses accurate spin-symmetric and spinantisymmetric static structure factors to build the local-field factors. In fact, the idea here is not entirely new, it has been used by Iwamoto et $a l .{ }^{37}$ in the context of 3D and by Dharma-wardana and Perrot ${ }^{38}$ in 2D electron liquids. For this purpose we implement the self-consistent Fermi hypernettedchain approach ${ }^{28-30}$ in order to calculate the spin-symmetric and spin-antisymmetric static structure factors incorporating the finite thickness effects in a quantum well. In what follows we first explain the FHNC approximation and then outline our method to obtain the static local-field factors $G_{ \pm}(q)$.

With the zero of energy taken at the chemical potential, the formally exact differential equation for the paircorrelation function $g_{\sigma \sigma^{\prime}}(r)$ reads

$$
\left[-\frac{\hbar^{2}}{m} \nabla_{\mathbf{r}}^{2}+v(r)+v_{\mathrm{P}}^{\sigma \sigma^{\prime}}(r)+V_{\mathrm{EKS}}^{\sigma \sigma^{\prime}}(r)\right] \sqrt{g_{\sigma \sigma^{\prime}}(r)}=0 .
$$

Here, the $v(r)$ is the Q2D potential that it is weaker than the Coulomb potential and its Fourier is given by $v_{\mathbf{q}}$ in $q$-space. The "Pauli potential" $v_{\mathrm{P}}^{\sigma \sigma^{\prime}}(r)$ is defined by ${ }^{39}$

$$
v_{\mathrm{P}}^{\sigma \sigma^{\prime}}(r)=\frac{\hbar^{2}}{m} \frac{\nabla_{\mathbf{r}}^{2} \sqrt{g_{\sigma \sigma^{\prime}}^{\mathrm{HF}}(r)}}{\sqrt{g_{\sigma \sigma^{\prime}}^{\mathrm{HF}}(r)}} .
$$

In Eq. (22), $g_{\sigma \sigma^{\prime}}^{\mathrm{HF}}(r)$ are the spin-resolved pair-correlation functions in the Hartree-Fock approximation (HF) which for the same spin is given by

$$
g_{\sigma \sigma}^{\mathrm{HF}}(r)=1-\left[\frac{2 J_{1}\left(k_{F}^{\sigma} r\right)}{k_{F}^{\sigma} r}\right]^{2},
$$

where $J_{1}(x)$ is the first-order Bessel function and $k_{F}^{\uparrow}$ $=k_{F} \sqrt{1+\zeta}, k_{F}^{\downarrow}=k_{F} \sqrt{1-\zeta}$. Moreover, $g_{\sigma \bar{\sigma}}^{\mathrm{HF}}(r)=1$. Although the expression for the Pauli potential is exact only for a weakly coupled $2 \mathrm{D} \mathrm{EG},{ }^{39}$ we shall assume in the following that it can yield useful results in our FHNC approach in Q2D EG.

The FHNC expresses the potential $V_{\mathrm{EKS}}^{\sigma \sigma^{\prime}}(r)$ in Eq. (21), which is the sum of the Hartree and of the exchangecorrelation potential, as the sum of two effective pair interactions: ${ }^{28-30}$

$$
V_{\mathrm{EKS}}^{\sigma \sigma^{\prime}}(r)=W_{\mathrm{B}}^{\sigma \sigma^{\prime}}(r)+\delta_{\sigma \sigma^{\prime}} W_{\mathrm{e}}^{\sigma \sigma}(r) .
$$

The first term on the left-hand side of Eq. (24) descends from the two-body correlation functions $u_{\sigma \sigma^{\prime}}(r)$ in the JastrowFeenberg wave function and is therefore formally the same 
as for a binary boson mixture. The second term is instead due to the antisymmetry of the fermion many-body wave function.

As shown by Chakraborty ${ }^{40}$ in treating a binary fermion mixture, the HNC closure yields for $u_{\sigma \sigma^{\prime}}(r)$ the expression

$$
u_{\sigma \sigma^{\prime}}(r)=\ln g_{\sigma \sigma^{\prime}}(r)-\left[g_{\sigma \sigma^{\prime}}(r)-1\right]+c_{\sigma \sigma^{\prime}}(r),
$$

where $c_{\sigma \sigma^{\prime}}(r)$ are the direct correlation functions, which are related to $g_{\sigma \sigma^{\prime}}(r)$ by the Ornstein-Zernike relations. ${ }^{41} \mathrm{We}$ introduce at this point the partial structure factors $S_{\sigma \sigma^{\prime}}(q)$ of the binary mixture, which in essence are the Fourier transforms of $g_{\sigma \sigma^{\prime}}(r)$ :

$$
S_{\sigma \sigma^{\prime}}(q)=\delta_{\sigma \sigma^{\prime}}+\sqrt{n_{\sigma^{\prime}} n_{\sigma^{\prime}}} \int d \mathbf{r}\left[g_{\sigma \sigma^{\prime}}(r)-1\right] \exp (-i \mathbf{q} \cdot \mathbf{r}) .
$$

We also introduce the Fourier transform of $W_{\mathrm{B}}^{\sigma \sigma^{\prime}}(r)$ $\left[W_{\mathrm{B}}^{\sigma \sigma^{\prime}}(q)\right.$, say]. Minimization of the ground state energy against arbitrary variations of $g_{\sigma \sigma^{\prime}}(r)$ yields with the help of Eq. (25) the expression

$$
W_{\mathrm{B}}^{\sigma \sigma^{\prime}}(q)=-\frac{\varepsilon_{q}}{\sqrt{n_{\sigma} n_{\sigma^{\prime}}}}\left[S_{\sigma \sigma^{\prime}}(q)-\delta_{\sigma \sigma^{\prime}}\right]-V_{\sigma \sigma^{\prime}}(q),
$$

where $\varepsilon_{q}=\hbar^{2} q^{2} /(2 m)$ are the single-particle kinetic energies and the functions $V_{\sigma \sigma^{\prime}}(q)$ that is the Fourier transform of "particle-hole" interaction, $V_{\sigma \sigma^{\prime}}(r)$ are given by

$$
\begin{aligned}
V_{\sigma \sigma^{\prime}}(r)= & g_{\sigma \sigma^{\prime}}(r)\left[v(r)+W_{\mathrm{e}}^{\sigma \sigma^{\prime}}(r)+v_{\mathrm{P}}^{\sigma \sigma^{\prime}}(r)\right] \\
& +\left[g_{\sigma \sigma^{\prime}}(r)-1\right] W_{\mathrm{B}}^{\sigma \sigma^{\prime}}(r)+\frac{\hbar^{2}}{m}\left|\nabla \sqrt{g_{\sigma \sigma^{\prime}}(r)}\right|^{2},
\end{aligned}
$$

and in general

$$
\begin{gathered}
S_{\sigma \sigma}(q)=\sqrt{A_{\bar{\sigma} \bar{\sigma}} \Delta^{2}-\frac{A_{\sigma \bar{\sigma}}^{2} \Delta^{3}}{2+\left[A_{\sigma \sigma}+A_{\bar{\sigma} \bar{\sigma}}\right] \Delta}}, \\
S_{\sigma \bar{\sigma}}(q)=\frac{A_{\sigma \bar{\sigma}} \Delta^{3 / 2}}{\sqrt{2+\left[A_{\sigma \sigma}+A_{\bar{\sigma} \bar{\sigma}}\right] \Delta}},
\end{gathered}
$$

where $\quad A_{\sigma \sigma^{\prime}}(q)=\delta_{\sigma \sigma^{\prime}}+2 \operatorname{sgn}\left(\sigma \sigma^{\prime}\right) \sqrt{n_{\sigma^{n}} n_{\sigma^{\prime}}} V_{\sigma \sigma^{\prime}}(q) / \varepsilon_{q} \quad$ and $\Delta(q)=\left(A_{\uparrow \uparrow} A_{\downarrow \downarrow}-A_{\uparrow \downarrow}^{2}\right)^{-1 / 2}$.

Turning to the second term on the left-hand side of Eq. (24), the effective pair potential $W_{\mathrm{e}}^{\sigma \sigma}(r)$ has a very complicated expression within the FHNC. ${ }^{28-30}$ However, in dealing with a one-component electron fluid Kallio and Piilo ${ }^{42}$ have proposed a simple and effective way to account for this consequence of the antisymmetry of the fermion wave function. Their argument is immediately generalized to our twocomponent Fermi fluid, and leads to the requirement that in Fourier transform this term should cancel the effective bosonlike interaction $W_{\mathrm{B}}^{\sigma \sigma}(q)$ for parallel-spin electrons at low coupling. That is

$$
W_{\mathrm{e}}^{\sigma \sigma}(q)=-\lim _{r_{s} \rightarrow 0} W_{\mathrm{B}}^{\sigma \sigma}(q)=\frac{\varepsilon_{q}}{2 n_{\sigma}}\left[1+2 S_{\sigma \sigma}^{\mathrm{HF}}(q)\right]\left[\frac{S_{\sigma \sigma}^{\mathrm{HF}}(q)-1}{S_{\sigma \sigma}^{\mathrm{HF}}(q)}\right]^{2} .
$$

Here, $S_{\sigma \sigma}^{\mathrm{HF}}(q)$ is the Hartree-Fock structure factor, which is given by

$$
\begin{aligned}
S_{\sigma \sigma}^{\mathrm{HF}}(q)= & \frac{2}{\pi}\left[\sin ^{-1}\left(\frac{q}{2 k_{F}^{\sigma}}\right)+\frac{q}{2 k_{F}^{\sigma}} \sqrt{1-\left(\frac{q}{2 k_{F}^{\sigma}}\right)^{2}}\right] \theta\left(2 k_{F}^{\sigma}-k\right) \\
& +\theta\left(2 k_{F}^{\sigma}-k\right) .
\end{aligned}
$$

It is evident that using Eqs. (26)-(29) requires a selfconsistent calculation of the spin-resolved static structure factors.

\section{Fluctuation-dissipation theorem}

The fluctuation-dissipation theorem which is of paramount importance for systems in equilibrium relates the dynamic susceptibilities defined above to the static structure factors

$$
S_{ \pm}(q)=-\frac{1}{n \pi} \int_{0}^{\infty} d \omega \operatorname{Im}\left[\chi_{C, S}(q, \omega)\right],
$$

where $S_{ \pm}(q)=\left[S_{\uparrow \uparrow} \pm S_{\uparrow \downarrow}\right] / 2$. As $\chi_{C}(q, \omega)$ and $\chi_{S}(q, \omega)$ depend on $G_{+}(q)$ and $G_{-}(q)$, respectively, the above integral expression allows one to determine the local-field factors once the static structure factors are calculated by the FHNC approach. The same approach of obtaining the local-field factors has previously been employed by Iwamoto $^{37}$ and Dharmawardana and Perrot. ${ }^{38}$ We note that the use of fluctuationdissipation theorem to extract static local-field factors is approximate in nature as it neglects the frequency dependence of $G_{ \pm}(q, \omega)$ from the outset. However, the apparent success of previous implementations ${ }^{37,38}$ encourages us to use it also in the present context.

As an additional simplification to the above procedure, one can further approximate the full $\chi_{0}(q, \omega)$ by the meanspherical approximation (MSA), viz.

$$
\chi_{0}^{\mathrm{MSA}}(q, \omega)=\frac{\left(n \hbar^{2} q^{2} / m\right)}{\omega^{2}-\left[\frac{\hbar^{2} q^{2}}{2 m S_{H F}(q)}\right]^{2}},
$$

in which $S_{H F}(q)$ is the Hartree-Fock static structure factor. With this approximation, the fluctuation-dissipation integral can be performed analytically to yield

$$
G_{+}(q)=1-\frac{\sqrt{2}}{4 r_{s}}\left(\frac{q}{k_{F}}\right)^{3} \frac{1}{F(q d)}\left[\frac{1}{S_{+}(q)^{2}}-\frac{1}{S_{H F}(q)^{2}}\right],
$$

and

$$
G_{-}(q)=-\frac{\sqrt{2}}{4 r_{s}}\left(\frac{q}{k_{F}}\right)^{3} \frac{1}{F(q d)}\left[\frac{1}{S_{-}(q)^{2}}-\frac{1}{S_{H F}(q)^{2}}\right] .
$$

Expressions similar to the above have been used in the context of charged boson fluids ${ }^{43}$ with the replacement of $S_{H F}(q)$ by unity. The MSA is essentially a plasmon-pole type ap- 


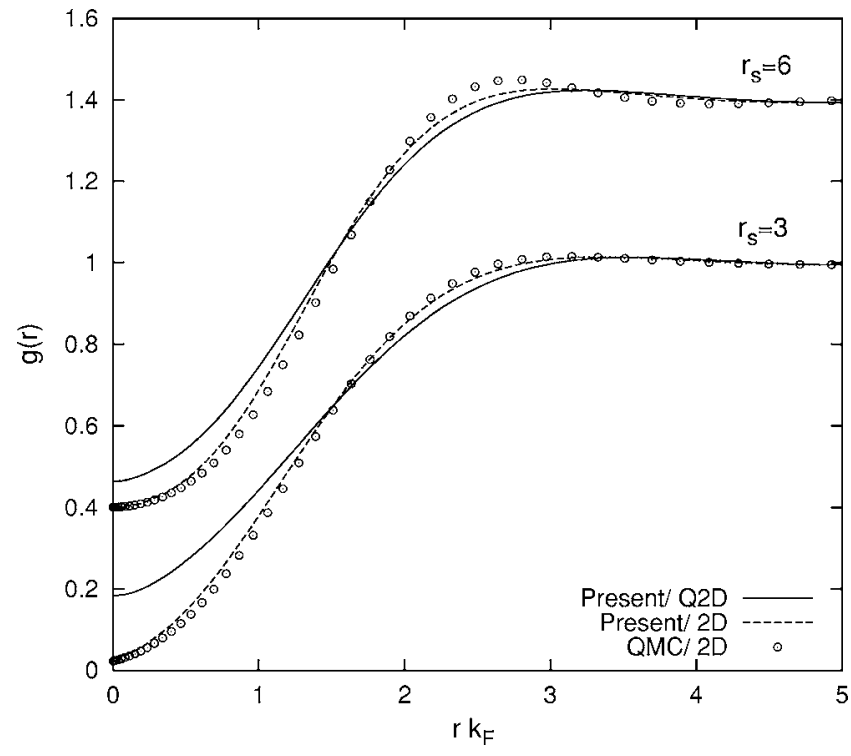

FIG. 1. Pair-correlation function $g(r)$ for $r_{s}=3$ (lower curves) and $r_{s}=6$ (upper curves have been shifted upwards by 0.4). Symbols are QMC results of Gori-Giorgi et al. (Ref. 44) for a strictly 2D electron gas, dashed and solid lines are those calculated within the present approach for 2D and Q2D EG, respectively.

proximation, where the particle-hole excitations are replaced by a single collective mode. We have found that it works quite well when the frequency integral in the fluctuationdissipation theorem is performed. The efficacy of MSA compared to using the full $\chi_{C}(q, \omega)$ and $\chi_{S}(q, \omega)$ in the fluctuation-dissipation integral is discussed in detail in our previous work. ${ }^{36}$

\section{NUMERICAL RESULTS}

We turn to the presentation of our numerical results, which are based on the local-field factors incorporating the quantum well finite thickness. We have numerically solved the FHNC set of equations, Eqs. (26)-(29), by repeating until self-consistency is achieved. We have calculated in this way the spin-resolved static structure factors of a Q2D EG. By implementing the static structure factors coming from the FHNC calculation in the fluctuation-dissipation theorem, ${ }^{36}$ we obtain the local-field factors.

To assess the validity of our procedure, we first show the calculated pair-correlation function $g(r)$ at two representative values of $r_{s}=3$ and $r_{s}=6$ in Fig. 1. For the strictly 2D system we compare our $g(r)$ with that obtained by Gori-Giorgi et $a l .{ }^{44}$ from QMC simulations. For these intermediate densities we find very good agreement and the omission of bridge diagrams within the FHNC is justified a posteriori. Figure 1 also shows our results for a Q2D EG. The effect of finite thickness on $g(r)$ is more appreciable for small values of $r$. Pair-correlation function $g(r)$ for a Q2D EG has also been calculated by Dharma-wardana ${ }^{27}$ within the $\mathrm{CHNC}$ approach which includes the bridge diagrams. We note that there are qualitative differences at small distances and in particular the on-top value $g(0)$. It is not clear at this point whether these
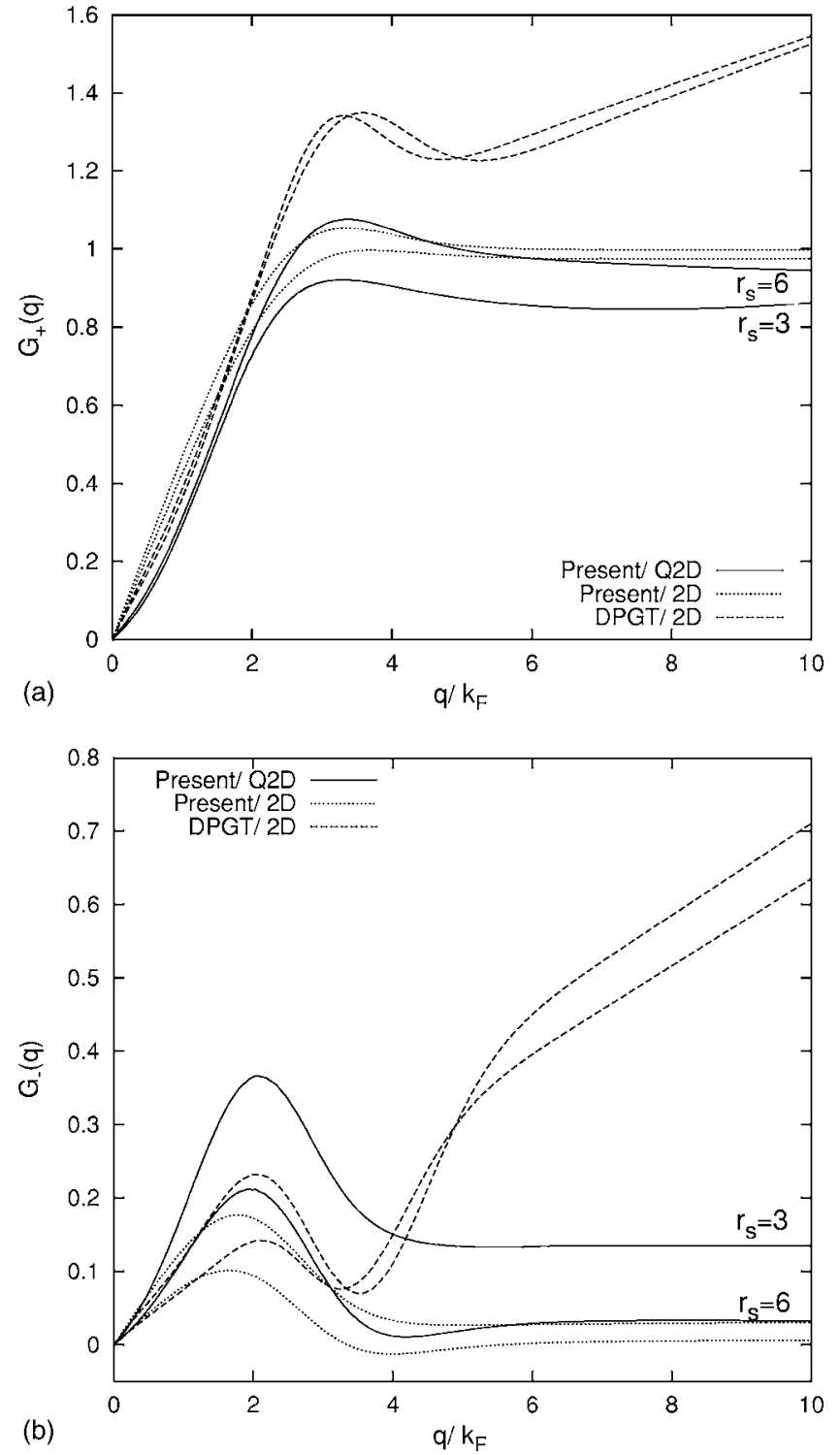

FIG. 2. Top: The local-field factors $G_{+}(q)$ as a function of $q / k_{F}$ for $r_{s}=3$ and $r_{s}=6$. Note that $G_{+}(q \rightarrow \infty)$ tends to a larger constant value with increasing $r_{s}$. Bottom: The local-field factors $G_{-}(q)$ as a function of $q / k_{F}$ for $r_{s}=3$ and $r_{s}=6$. In both figures, dashed lines are analytical expression of QMC results of Davoudi et al. (Ref. 45) for a strictly $2 \mathrm{D}$ electron gas, dotted and solid lines are those calculated within the present approach for 2D and Q2D EG, respectively. Note that $G_{-}(q \rightarrow \infty)$ tends to a smaller constant value with increasing $r_{s}$.

differences are because of the neglect of bridge functions in our implementation of the FHNC calculation or not. QMC simulations for Q2D EG would help establish the correct behavior of $g(r)$ for finite thickness samples.

We next display the local-field factors $G_{+}(q)$ and $G_{-}(q)$ calculated within the present approach in comparison to those constructed by Davoudi et al. ${ }^{45}$ using the QMC data and known sum-rules. Our local-field factors typically start at zero in the long-wavelength limit and go to a constant for large values of $q$ as shown in Fig. 2. The main qualitative difference between our $G_{+}(q)$ and $G_{-}(q)$ and the Davoudi et 


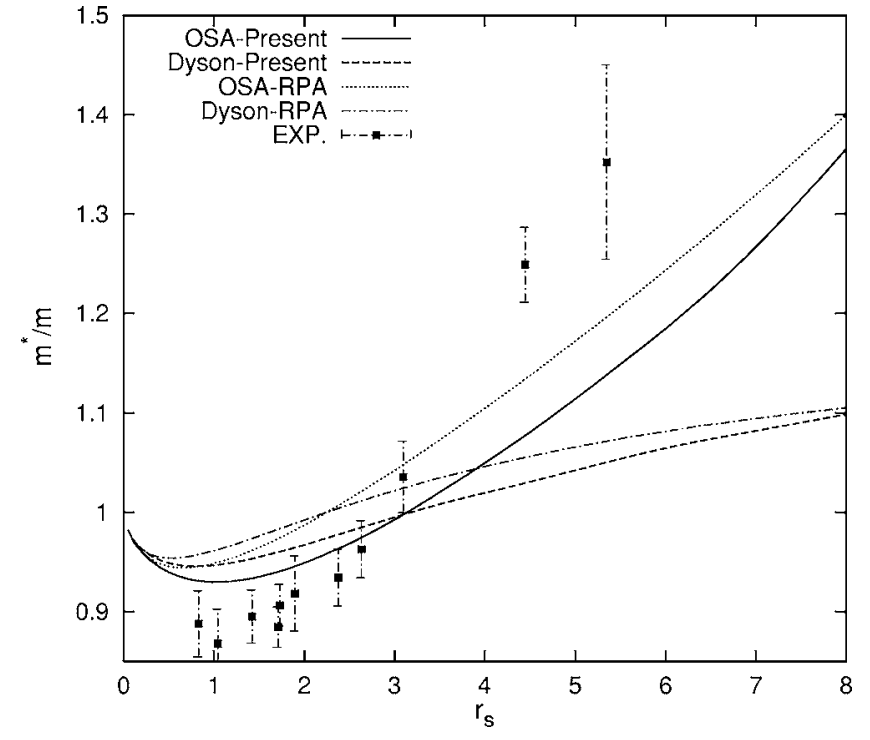

FIG. 3. Many-body effective mass as a function of $r_{s}$ for $0 \leqslant r_{s} \leqslant 8$ for a quasi-2D EG confined in a GaAs/AlGaAs triangular quantum well of the type used in Ref. 12.

$a l .{ }^{45}$ construction is in the large $q$ behavior. In particular, a peak structure both in $G_{+}(q)$ and $G_{-}(q)$ occurring around $q$ $\approx 3 k_{F}$ and $q \approx 2 k_{F}$, respectively, is quite well reproduced. Our local-field factors satisfy the fluctuation-dissipation theorem but not the compressibility sum-rule, whereas those of Davoudi et al. ${ }^{45}$ satisfy the compressibility sum-rule but not the fluctuation dissipation theorem. In fact, it is known that frequency dependent (dynamical) local-field factors are needed to fulfill both requirements.

We use thus obtained local-field factors to calculate the effective mass, modified Landé $g^{*}$-factor, and the spinsusceptibility of a Q2D EG. Comparison of the local-field factors for $2 \mathrm{D}$ and $\mathrm{Q} 2 \mathrm{D}$ systems in the previous figures reveals the fact that there are quantitative differences due to the finite thickness effect.

In Fig. 3 we show our numerical results of the QP effective mass both in OSA and Dyson approximations. The QP effective mass enhancement is substantially smaller in the Dyson equation calculation than in the OSA, the reason being that a large cancellation occurs between the numerator and the denominator in Eq. (13). To clarify the effect of charge- and spin-density fluctuations we have also included the RPA results which do not take the spin fluctuations into account. Comparing the results of Fig. 3 with the experimental measurements of Tan et al. ${ }^{12}$ we can draw the following conclusions: (i) The RPA and present results are rather similar in the weak coupling limit $\left(r_{s} \ll 1\right)$, and (ii) theoretical calculations in the strong coupling region are not so close to experimental data. There is an essential point which we should stress here that experimental data were collected at weak magnetic fields and mostly in high Landau levels, however, our numerical calculations have been performed in the absence of a magnetic field.

In Fig. 4 we show the finite thickness effect of Q2D EG compared to a 2D EG. As it is clear from the figure the QP effective mass in both OSA and Dyson calculations, the ef-

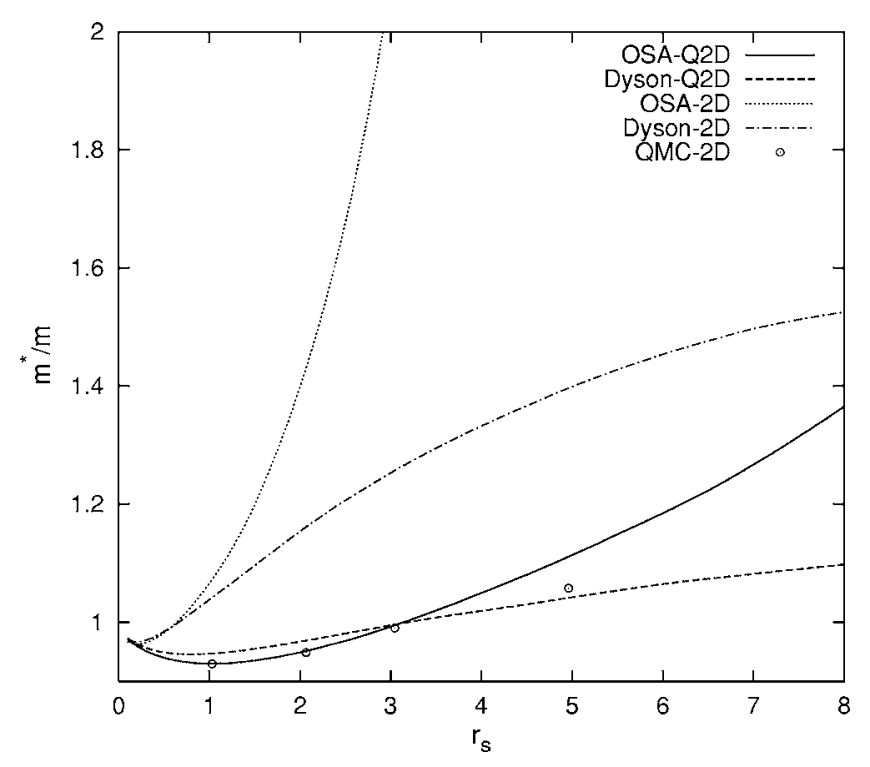

FIG. 4. Many-body effective mass as a function of $r_{s}$ for $0 \leqslant r_{s} \leqslant 8$ for quasi-2D EG in comparison to 2D EG. Symbols are QMC results of Kwon et al. (Ref. 46) for a strictly 2D electron gas.

fect of finite thickness of the quantum well reduces the QP effective mass from the strictly $2 \mathrm{D}$ results. The reason for this is understandable because the strength of bare quasi-2D potential is reduced by the form factor in Q2D EG. For comparison we have also included in this figure the variational QMC results of Kwon et al. ${ }^{46}$ for a 2D EG. Our corresponding calculations (for a 2D EG) within the Dyson approximation are not in agreement with QMC results. However, the reader should bear in mind that the effective mass is not a ground-state property and thus its evaluation by the QMC technique is quite delicate, as it involves the construction of excited states.

It has been noted in the literature ${ }^{19,20}$ that there is a divergence in the QP effective mass near $r_{s} \approx 5$ in $2 \mathrm{D}$ EG within the OSA and this divergence occurs at $r_{s} \approx 15.5$ when the self-energy is evaluated in the RPA/OSA. We find similar divergent behavior in $m^{*}$ for Q2D when OSA is used. The unphysical nature of this divergence has been discussed in detail in Ref. 22. Briefly, Eq. (15) is a valid approximation to the effective mass in the weak coupling limit, as can be seen by expanding Eq. (13) for small values of $\sum_{\text {ret }}^{\mathrm{R}}$ : however, its application becomes problematic at large values of $r_{s}$. In particular, we see that because $-\partial_{\omega} \operatorname{Re} \sum_{\text {ret }}^{\mathrm{R}}\left(k_{F}, 0\right)$ increases monotonically with increasing $r_{s}$, there must necessarily be a critical value of $r_{s}$ for which the denominator of Eq. (15) vanishes and $m_{\mathrm{OSA}}^{*}$ diverges. In our view, however, this must be considered an artifact of Eq. (15). Its unphysical character is revealed by the fact that the divergence is driven by a negative but finite value of $\partial_{\omega} \operatorname{Re} \Sigma_{\text {ret }}^{\mathrm{R}}\left(k_{F}, 0\right)$, whereas we know, from the general analysis, that a genuine divergence would have to be driven either by an infinite $\partial_{\omega} \operatorname{Re} \sum_{\text {ret }}^{\mathrm{R}}\left(k_{F}, 0\right)$ or by a negative $\partial_{k} \operatorname{Re} \sum_{\text {ret }}^{\mathrm{R}}\left(k_{F}, 0\right)$ becoming equal to $-\hbar^{2} k_{F} / m$. We conclude that there is no evidence, within the present theory, for a divergence of the effective mass.

We have computed the QP excitation energy using Eq. (10) and present our results in Fig. 5. In this figure we show 


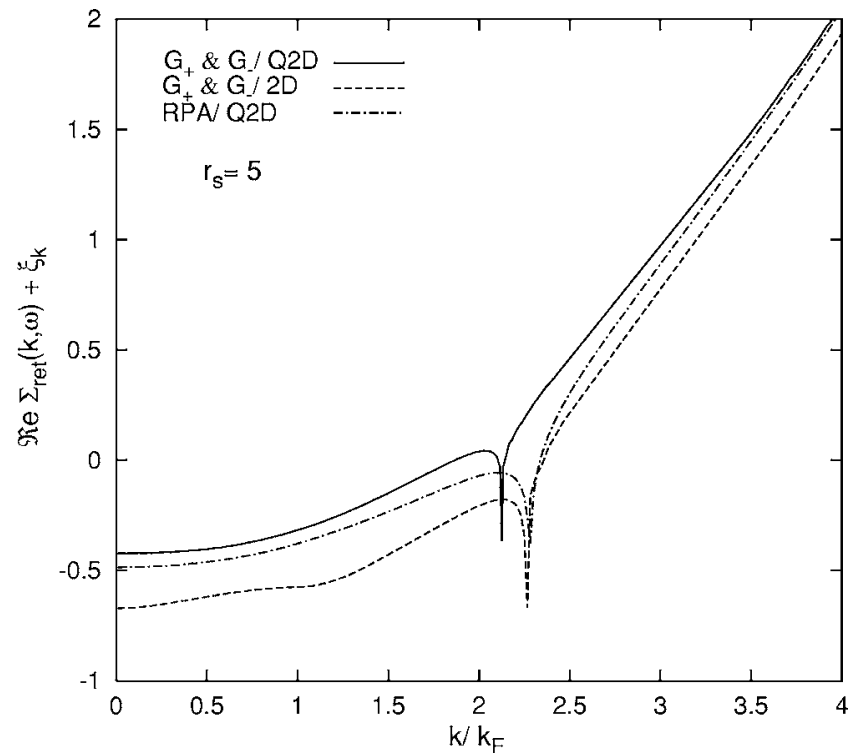

FIG. 5. The real part of retarded self-energy plus $\xi_{k}$ evaluated at $\omega=\xi_{k} / \hbar$ as a function of $k / k_{F}$ for $r_{s}=5$.

the real part of retarded self-energy plus $\xi_{k}$ evaluated at the single-particle frequency $\omega(k)=\xi_{\mathbf{k}} / \hbar$ at $r_{s}=5$. Note the presence of a strong dip at a particular value of $k\left(k_{c}\right.$, for instance) which depends on $r_{s}$, finite size effect, and on the functional form of the charge-charge local-field factor. This is the plasmon dip, which is also present in three dimensions ${ }^{47}$ and originates from the fact that at each $r_{s}$ there is a sufficiently high value of $k$ for the decay of an electronhole pair into a plasmon with conservation of momentum and energy. Another important issue here is the flatness of the real part of retarded self-energy for a 2D EG at $k=k_{F}$. This structure implies a divergent behavior in $m^{*}(k)$ at $k=k_{F}$. It is interesting to note in Fig. 5 that when finite thick-

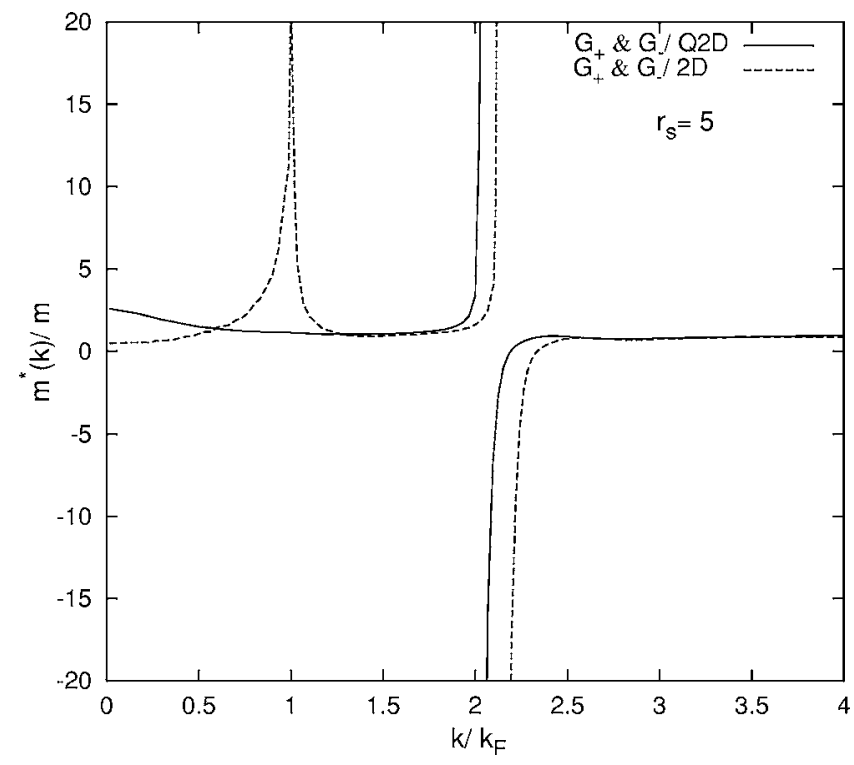

FIG. 6. Many-body on-shell effective mass as a function of $k / k_{F}$ at $r_{s}=5$ for Q2D EG with the combined effect of charge and spin fluctuations in comparison to 2D EG.

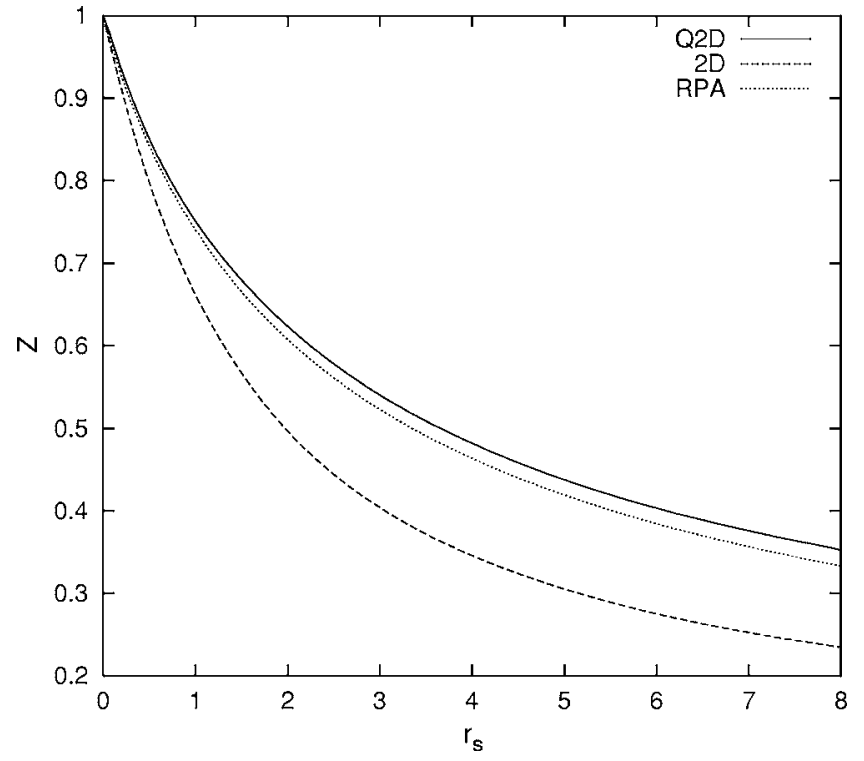

FIG. 7. Renormalization constant $Z$ as a function of $r_{s}$ for $0 \leqslant r_{s} \leqslant 8$.

ness effects are included the flatness in the real part of selfenergy at $k=k_{F}$ is lifted.

To gain further insight on the density dependence of the effective mass we have also calculated the on-shell effective mass as a function of particle momentum $k$ using Eq. (11) evaluated at $\omega(k)=\xi_{\mathbf{k}} / \hbar$ at $r_{s}=5$. The results are shown in Fig. 6. The $m^{*}(k)$ is essentially very weakly momentum dependent for $k<k_{F}$ for a Q2D EG. There is a substantial cancellation between the residue and the exchange plus line selfenergy contribution in this regime for these cases which make the real part of retarded self-energy approximately linear with respect to $k$. As we mentioned above, the presence of a divergence in the effective mass at a value of $k_{c}$ which depends on $r_{s}$, finite size effect, and on the functional form of the charge-charge local field factor is due to the plasmon dip. However, in the 2D EG case there is a peak at $k=k_{F}$ which is below the plasmon threshold as also noted by $\mathrm{Ng}$ and Singwi. ${ }^{47}$ Similar calculations within the RPA have recently been reported by Zhang et al. ${ }^{21}$ where they have studied in detail the divergence in $m^{*}(k)$. We find that such a divergent peaked structure disappears when finite thickness effects are included.

In Fig. 7 we show our numerical calculations for the renormalization constant $Z$ as a function of $r_{s}$. The effects of charge- and spin-fluctuations make the $Z$ values bigger than the results when these effects are not included. Furthermore, the finite size quantum well also makes the $Z$ values bigger than the results of $2 \mathrm{D} \mathrm{EG}$ as well. That the renormalization constant displays a smooth behavior as a function of $r_{s}$ may be taken as an indication of the Fermi liquid picture being preserved for this density regime.

Figure 8 depicts our results for the ratio $g^{*} / g$ as a function of $r_{s}$ for $0 \leqslant r_{s} \leqslant 6 . g^{*} / g$ is calculated from Eq. (16) and embodies the charge and spin fluctuation effects through $G_{+}$ and $G_{-}$. We included the value of experimental $\chi^{*} m / \chi_{0} m^{*}$ which is extracted from the $\chi^{*} / \chi_{0}$ empirical formula given by Tan et al. ${ }^{11}$ divided by the experimental data of $\mathrm{m}^{*} / \mathrm{m}$ of 


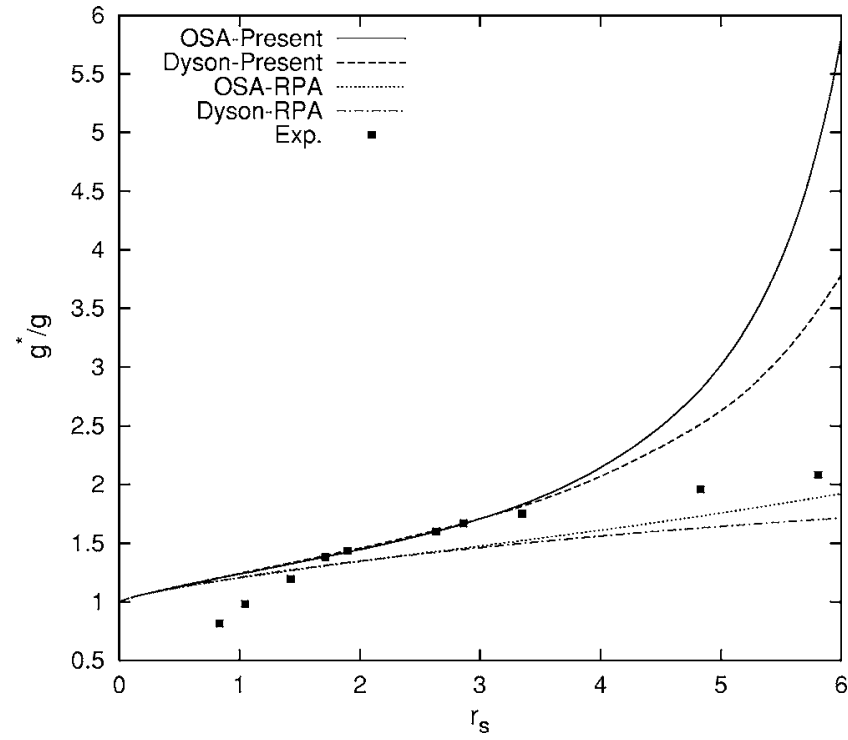

FIG. 8. Plot of the ratio $g^{*} / g$ as a function of $r_{s}$ for $0 \leqslant r_{s} \leqslant 6$. The experimental data $\chi^{*} \mathrm{~m} / \chi_{0} m^{*}$ is from the $\chi^{*} / \chi_{0}$ of empirical formula given by Tan et al. (Ref. 11) divided by the $\mathrm{m}^{*} / \mathrm{m}$ of Tan $e t$ al. experimental data (Ref. 12).

Tan et al. ${ }^{12}$ We observe that there is an enhancement in $g^{*}$ beyond $r_{s} \sim 5$ within the present method using either OSA or the Dyson approaches compared to the experimental data and the RPA calculation. In particular, it is surprising that RPA yields a reasonable agreement with experiment in a region of $r_{s}$ values where it is not expected to be very reliable.

To understand the enhancement in $g^{*}$, we show in Fig. 9 the behavior of the two terms in the denominator of Eq. (16) as functions of $r_{s}$. This figure clearly illustrates how a divergence can arise in $g^{*}$ both in OSA and Dyson approximations. For instance, within the OSA the denominator in Eq.

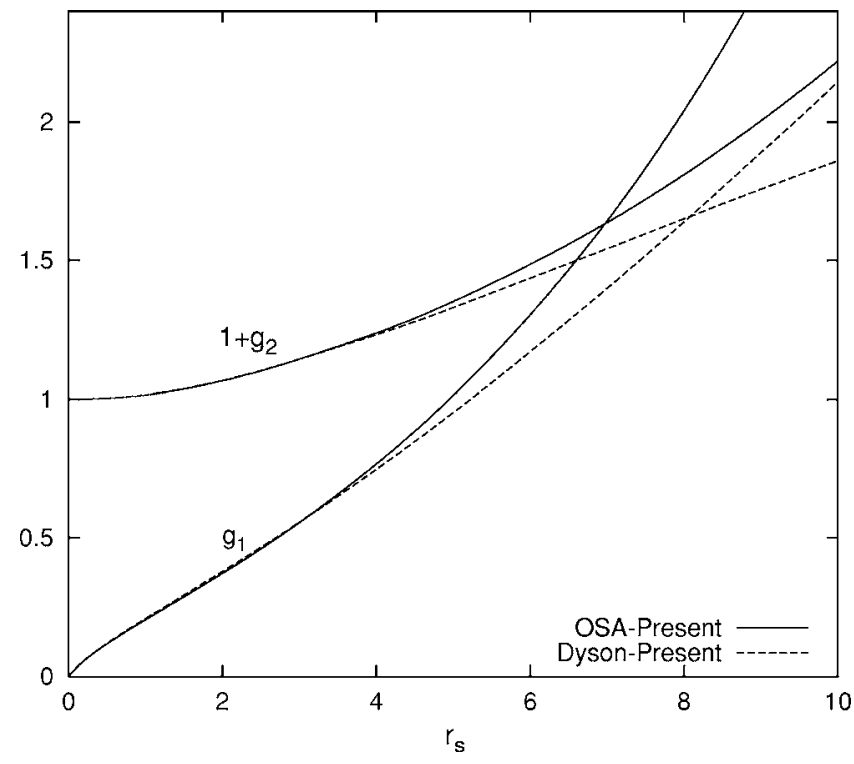

FIG. 9. Illustrating the divergence of the modified Landé $g^{*}$-factor within the OSA and Dyson approximations. The two curves starting from unity at $r_{s}=0$ refer to the quantity $1+g_{2}$ and the other two curves to $g_{1}$.

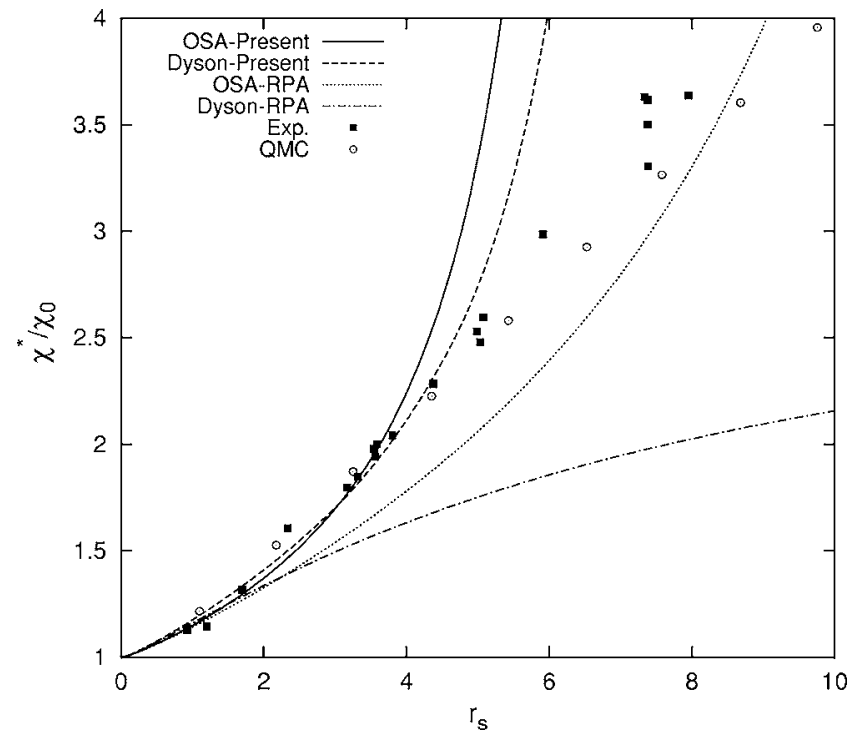

FIG. 10. Many-body spin susceptibility as a function of $r_{s}$ for $0 \leqslant r_{s} \leqslant 10$ for a Q2D EG confined in a GaAs/AlGaAs triangular quantum well of the type used in Ref. 11 compared with quantum Monte Carlo results (Ref. 26).

(16) has a zero around $r_{s} \approx 7$ and $r_{s} \approx 8$ within the Dyson approximation.

In Fig. 10 we show the spin susceptibility as a function of $r_{s}$ compared to RPA, recent experimental data of Zhu et al., ${ }^{11}$ and quantum Monte Carlo calculation. ${ }^{26}$ As it is clear from this figure $\chi^{*} / \chi$ starts at unity when $r_{s}$ tends to zero and increases with increasing $r_{s}$ values. Our numerical calculations within both OSA and Dyson approximations are in good agreement with the experimental measurements in the weak and intermediate coupling limits. To see more clearly the effect of finite thickness of a quantum well, we have shown the spin susceptibility both in Q2D EG and 2D EG in

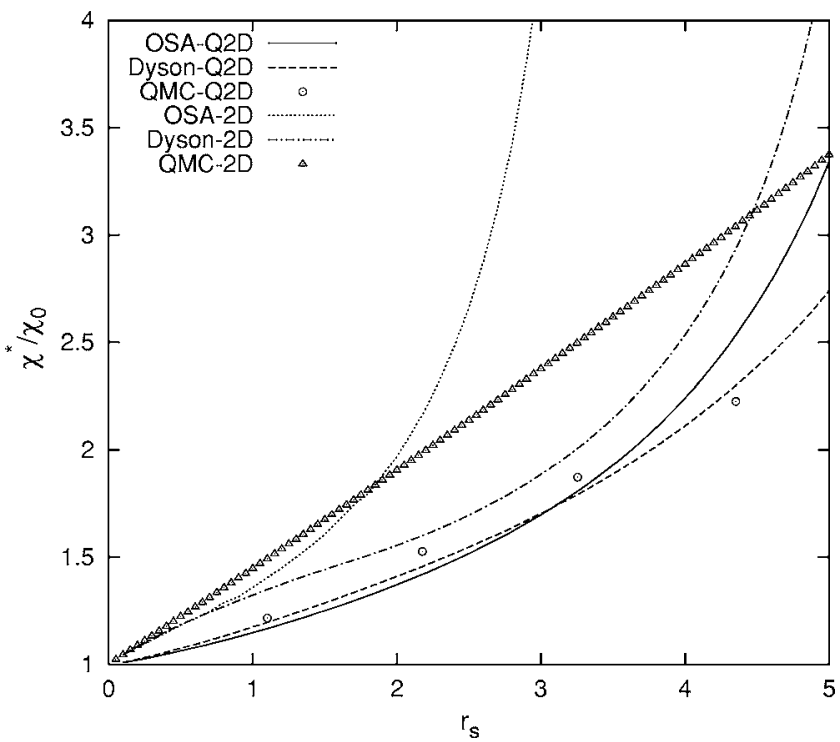

FIG. 11. Many-body spin susceptibility as a function of $r_{s}$ for $0 \leqslant r_{s} \leqslant 10$ for both Q2D EG and 2D EG which are compared with quantum Monte Carlo results (Refs. 26 and 15). 
comparison to the QMC data of Refs. 26 and 15 in Fig. 11. Our numerical calculations are in better agreement with the QMC compared to the 2D EG case.

The above results for the spin susceptibility basically reflect the present status of the perturbation theory based calculations despite the fact that a lot of effort has been expanded to improve the quasiparticle interactions. The poor agreement with experimental data beyond $r_{s} \sim 4$ appears to be associated with the shortcomings of the formalism. Our calculations indicate that finite thickness effects alone cannot account for the discrepancy. It would be important to improve upon this outstanding theoretical problem. The recent QMC calculation by De Palo et al. ${ }^{26}$ which represents the experiments quite well, on the other hand, is based on the accurate evaluation of the ground-state energy and therefore is of a different nature than our approach. It is of theoretical interest to bring the level of agreement between different approaches closer.

\section{SUMMARY}

We have presented a study of the effect of many-body charge- and spin-fluctuations in a quasi-two-dimensional electron liquid using the Fermi hypernetted chain approach to build the local-field factors incorporating the finite thickness of the quantum well. In particular, we used the sample parameters of the structure used in the experiments of Zhu et al. ${ }^{11}$ and Tan et al. ${ }^{12}$ We have carried out extensive calculations of the retarded quasiparticle self-energy which use the finite thickness dependent local-field factors as input. We have presented our results of the quasiparticle effective mass, many-body renormalization constant, modified Landé $g^{*}$-factor, and spin susceptibility in a GaAs/AlGaAs triangular quantum well over a wide range of the coupling strengths. The comparison with the experimental data of Refs. 12 and 11 shows that the simultaneous inclusion of charge- and spin-density fluctuations beyond the random phase approximation and essentially including the finite size quantum well effect is important to obtain reasonable agreement for $r_{s}$ $\lesssim 4$ between the experimental and theoretical quasiparticle transport properties. For larger values of $r_{s}$ the agreement with experiments is rather poor due to the approximate nature of the perturbation theory, and it remains a challenge to improve the quality of quasiparticle properties. There are several aspects by which the theoretical calculations may be improved quantitatively for a better agreement with experimental measurements. (1) Experimental data for the effective mass were collected in small magnetic fields, hence one needs to generalize our theory to take into account a magnetic field. (2) It is clear that the FHNC approximation works well for small and intermediate coupling strengths in 2D $\mathrm{EG}^{43}$ and one needs to go beyond the simple FHNC approximation by incorporating the bridge functions and triplet correlation functions. ${ }^{48}$

\section{ACKNOWLEDGMENTS}

We thank Y.-W. Tan and J. Zhu for providing us with their experimental data. B. T. acknowledges partial support from the Science and Technological Council of Turkey (TUBITAK) and Turkish Academy of Sciences (TUBA).
${ }^{1}$ T. Ando, A. B. Fowler, and F. Stern, Rev. Mod. Phys. 54, 437 (1982)

${ }^{2}$ G. F. Giuliani and G. Vignale, Quantum Theory of the Electron Liquid (Cambridge University Press, Cambridge, England, 2005).

${ }^{3}$ E. Abrahams, S. V. Kravchenko, and M. P. Sarachik, Rev. Mod. Phys. 73, 251 (2001); S. V. Kravchenko and M. P. Sarachik, Rep. Prog. Phys. 67, 1 (2004).

${ }^{4}$ V. T. Dolgopolov, G. V. Kravchenko, A. A. Shashkin, and S. V. Kravchenko, JETP Lett. 55, 733 (1992); D. Simonian, S. V. Kravchenko, M. P. Sarachik, and V. M. Pudalov, Phys. Rev. Lett. 79, 2304 (1997); V. M. Pudalov, G. Brunthaler, A. Prinz, and G. Bauer, JETP Lett. 65, 932 (1997).

${ }^{5}$ T. Okamoto, K. Hosoya, S. Kawaji, and A. Yagi, Phys. Rev. Lett. 82, 3875 (1999).

${ }^{6}$ S. A. Vitkalov, H. Zheng, K. M. Mertes, M. P. Sarachik, and T. M. Klapwijk, Phys. Rev. Lett. 87, 086401 (2001).

${ }^{7}$ A. A. Shashkin, S. V. Kravchenko, V. T. Dolgopolov, and T. M. Klapwijk, Phys. Rev. Lett. 87, 086801 (2001); Phys. Rev. B 66, 073303 (2002).

${ }^{8}$ V. M. Pudalov, M. E. Gershenson, H. Kojima, N. Butch, E. M. Dizhur, G. Brunthaler, A. Prinz, and G. Bauer, Phys. Rev. Lett. 88, 196404 (2002).

${ }^{9}$ E. Tutuc, S. Melinte, and M. Shayegan, Phys. Rev. Lett. 88, 036805 (2002).
${ }^{10}$ H. Noh, M. P. Lilly, D. C. Tsui, J. A. Simmons, E. H. Hwang, S. Das Sarma, L. N. Pfeiffer, and K. W. West, Phys. Rev. B 68, 165308 (2003).

${ }^{11}$ J. Zhu, H. L. Stormer, L. N. Pfeiffer, K. W. Baldwin, and K. W. West, Phys. Rev. Lett. 90, 056805 (2003); Y.-W. Tan, J. Zhu, H. L. Stormer, L. N. Pfeiffer, K. W. Baldwin, and K. W. West, Phys. Rev. B 73, 045334 (2006).

${ }^{12}$ Y.-W. Tan, J. Zhu, H. L. Stormer, L. N. Pfeiffer, K. W. Baldwin, and K. W. West Phys. Rev. Lett. 94, 016405 (2005).

${ }^{13}$ E. Tutuc, S. Melinte, E. P. De Poortere, M. Shayegan, and R. Winkler, Phys. Rev. B 67, 241309(R) (2003).

${ }^{14}$ K. Vakili, Y. P. Shkolnikov, E. Tutuc, E. P. De Poortere, and M. Shayegan, Phys. Rev. Lett. 92, 226401 (2004).

${ }^{15}$ C. Attaccalite, S. Moroni, P. Gori-Giorgi, and G. B. Bachelet, Phys. Rev. Lett. 88, 256601 (2002).

${ }^{16}$ L. D. Landau, Sov. Phys. JETP 3, 920 (1957).

${ }^{17}$ I. K. Marmorkos and S. Das Sarma, Phys. Rev. B 44, 3451 (1991); J. D. Lee and B. I. Min, ibid. 53, 10988 (1996); H.-J. Schulze, P. Schuck, and N. Van Giai, ibid. 61, 8026 (2000).

${ }^{18}$ S. Yarlagadda and G. F. Giuliani, Phys. Rev. B 49, 7887 (1994); 61, 12556 (2000); C. S. Ting, T. K. Lee, and J. J. Quinn, Phys. Rev. Lett. 34, 870 (1975).

${ }^{19}$ H. M. Böhm and K. Schörkhuber, J. Phys.: Condens. Matter 12, 2007 (2000).

${ }^{20}$ Y. Zhang and S. Das Sarma, Phys. Rev. B 71, 045322 (2005); S. 
Das Sarma, V. M. Galitski, and Y. Zhang, ibid. 69, 125334 (2004).

${ }^{21}$ Y. Zhang, V. M. Yakovenko, and S. Das Sarma, Phys. Rev. B 71, 115105 (2005).

${ }^{22}$ R. Asgari, B. Davoudi, M. Polini, G. F. Giuliani, M. P. Tosi, and G. Vignale, Phys. Rev. B 71, 045323 (2005).

${ }^{23}$ R. Asgari, B. Davoudi, and B. Tanatar, Solid State Commun. 130, 13 (2004).

${ }^{24}$ V. T. Dolgopolov, JETP Lett. 76, 377 (2002).

${ }^{25}$ B. Spivak, Phys. Rev. B 64, 085317 (2001).

${ }^{26}$ S. De Palo, M. Botti, S. Moroni, and G. Senatore Phys. Rev. Lett. 94, 226405 (2005).

${ }^{27}$ M. W. C. Dharma-wardana, Phys. Rev. B 72, 125339 (2005); Solid State Commun. 136, 76 (2005).

${ }^{28}$ L. J. Lantto and P. J. Siemens, Nucl. Phys. A 317, 55 (1979); L. J. Lantto, Phys. Rev. B 36, 5160 (1987).

${ }^{29}$ J. G. Zabolitzky, Phys. Rev. B 22, 2353 (1980).

${ }^{30}$ For a recent review see E. Krotscheck and M. Saarela, Phys. Rep. 232, 1 (1993).

${ }^{31}$ A. V. Chaplik, Sov. Phys. JETP 33, 997 (1971); see also F. Stern, Phys. Rev. Lett. 30, 278 (1973).

${ }^{32}$ C. A. Kukkonen and A. W. Overhauser, Phys. Rev. B 20, 550 (1979).

${ }^{33}$ F. Stern, Phys. Rev. Lett. 18, 546 (1967).

${ }^{34}$ G. S. Atwal, I. G. Khalil, and N. W. Ashcroft, Phys. Rev. B 67, 115107 (2003).
${ }^{35}$ Z. Qian and G. Vignale, Phys. Rev. B 65, 235121 (2002); 71, 169904(E) (2005).

${ }^{36}$ R. Asgari, A. L. Subaşı, A. A. Sabouri-Dodaran, and B. Tanatar (unpublished).

${ }^{37}$ N. Iwamoto, E. Krotscheck, and D. Pines, Phys. Rev. B 29, 3936 (1984).

${ }^{38}$ M. W. C. Dharma-wardana and F. Perrot, Europhys. Lett. 63, 660 (2003).

${ }^{39}$ B. Davoudi, R. Asgari, M. Polini, and M. P. Tosi, Phys. Rev. B 68, 155112 (2003).

${ }^{40}$ T. Chakraborty, Phys. Rev. B 25, 3177 (1982); 26, 6131 (1982); T. Chakraborty, A. Kallio, L. J. Lantto, and P. Pietiläinen, ibid. 27, 3061 (1983).

${ }^{41}$ L. S. Ornstein and F. Zernike, Phys. Z. 19, 134 (1918).

${ }^{42}$ A. Kallio and J. Piilo, Phys. Rev. Lett. 77, 4237 (1996).

${ }^{43}$ V. Apaja, J. Halinen, V. Halonen, E. Krotscheck, and M. Saarela, Phys. Rev. B 55, 12925 (1997).

${ }^{44}$ P. Gori-Giorgi, S. Moroni, and G. B. Bachelet, Phys. Rev. B 70, 115102 (2004).

${ }^{45}$ B. Davoudi, M. Polini, G. F. Giuliani, and M. P. Tosi, Phys. Rev. B 64, 153101 (2001); 64, 233110 (2001).

${ }^{46}$ Y. Kwon, D. M. Ceperley, and R. M. Martin, Phys. Rev. B 50, 1684 (1994).

${ }^{47}$ T.-K. Ng and K. S. Singwi, Phys. Rev. B 34, 7743 (1986).

${ }^{48}$ R. Asgari, B. Davoudi, and M. P. Tosi, Solid State Commun. 131, 130 (2004). 